\title{
The Index of Invariant Subspaces of Bounded below Operators on Banach Spaces
}

\author{
George Chailos \\ Department of Computer Science, University of Nicosia, Nicosia, Cyprus \\ Email: chailos.g@unic.ac.cy
}

Received November 17, 2011; revised November 30, 2011; accepted December 8, 2011

\begin{abstract}
For an operator $S$ on a Banach space $X$, let $\operatorname{Lat}(S, X)$ be the collection of all its invariant subspaces. We consider the index function on $\operatorname{Lat}(S, X)$ and we show, amongst others, that if $S$ is a bounded below operator and if $M_{i} \in \operatorname{Lat}(S, X), \quad i \in I \subset \mathbb{N}$, then $\sum_{i \in I}$ ind $M_{i} \geq \operatorname{ind}\left(\bigcap_{i \in I} M_{i}\right)+\operatorname{ind}\left(\bigvee_{i \in I} M_{i}\right)$. If in addition $M_{i}$ are index 1 invariant subspaces of $S$, with nonzero intersection, we show that ind $\left(\vee_{i \in I} M_{i}\right)<\operatorname{ind}\left(\sum_{i \in I} M_{i}\right)$. Furthermore, using the index function, we provide an example where for some $M_{i} \in \operatorname{Lat}(S, X)$, holds $\vee_{i \in I} M_{i}=\bigoplus_{i \in I} M_{i}$.
\end{abstract}

Keywords: Index; Invariant Subspaces; Bounded below Operators; Banach Spaces

\section{Introduction, the Index Function}

If $S$ is an operator on a Banach space $X$, then a closed subspace $M$ of $X$ is called invariant for $S$ if $S M \subset M$. The collection of all invariant subspaces of an operator $S$ is denoted by $\operatorname{Lat}(S, X)$. It forms a complete lattice with respect to intersections and closed spans. One of the important notions in the general theory of operators, such as bounded below operators, is the index of an element in $\operatorname{Lat}(S, X)$, which is defined as follows. (This definition is taken from [1].)

Definition 1.1. The map

$$
\text { ind }: \operatorname{Lat}(S, X) \rightarrow\{0\} \cup \mathbb{N} \cup\{\infty\}
$$

is defined as ind $M=\operatorname{dim}(M / S M)$ and ind $M=0$ if and only if $M=\{0\}$. We say that $M$ has index $n$ if ind $M=n$.

The index function plays an essential role in the study of invariant subspaces of Banach spaces. (For example, see an extensive study in [2] for index 1 invariant subspaces in Banach spaces of analytic functions.) In this article we generalize and extend the results obtained in [3], utilizing new proving techniques, deriving algebraic properties of the index functions. Moreover, we provide new results that are applied in Bergman space theory. Amongst others, and as a corollary to our main result, we show that if $M_{i} \in \operatorname{Lat}(S, X)$, ind $M_{i}=1$ and $\left(\bigcap_{i \in I} M_{i}\right) \neq\{0\}$ then ind $\left(\vee_{i \in I} M_{i}\right)<$ ind $\left(\sum_{i \in I} M_{i}\right)$, where $\vee_{i \in I} M_{i}$ denotes the closed span of $M_{i}$, $i \in I \subset \mathbb{N}$. (Equivalently, $\vee_{i \in I} M_{i}$ is the closure of $\left.\sum_{i \in I} M_{i}\right)$. An analogous result, but in not such a general setting as the one presented here, was proved by Richter ([2], Corollary 3.12), using operator theoretical tools and results from analysis. Here we prove our general result using only algebraic tools and a rather standard result from functional analysis. Furthermore, we provide an example where for some $M_{i} \in \operatorname{Lat}(S, X)$, $\vee_{i \in I} M_{i}=\bigoplus_{i \in I} M_{i}$ holds, and we present an application of this result in Bergman space theory.

\section{Algebraic Properties of the Index Function-Main Results}

In the sequel we denote with $I$ an index subset of $\mathbb{N}$, and with $X$ a Banach space.

Theorem 2.1. Let $\mathbf{R}$ be a commutative ring with identity and let $A, A_{i}$ be free unitary $\mathbf{R}$-modules such that $A_{i}$ are free submodules of $A, i \in I$. Then

$$
\sum_{i \in I} \operatorname{rank}\left(A / A_{i}\right)=\operatorname{rank}\left(A /\left(\bigcap_{i \in I} A_{i}\right)\right)+\operatorname{rank}\left(A / \sum_{i \in I} A_{i}\right) .
$$

Proof. We shall prove this theorem using mathe- matical induction. Henceforth at first we establish the following equation (which it is the initial step of mathematical induction.) We supposes that $A^{\prime}, B^{\prime}$ are free unitary $\mathbf{R}$-modules that are free submodules of $A$, then 


$$
\begin{aligned}
& \operatorname{rank}\left(A / A^{\prime}\right)+\operatorname{rank}\left(A / B^{\prime}\right) \\
& =\operatorname{rank}\left(A /\left(A^{\prime} \cap B^{\prime}\right)\right)+\operatorname{rank}\left(A /\left(A^{\prime}+B^{\prime}\right)\right)
\end{aligned}
$$

To prove $(\star)$, consider the following sequence

$$
0 \rightarrow A /\left(A^{\prime} \cap B^{\prime}\right) \stackrel{f}{\rightarrow} A / A^{\prime} \oplus A / B^{\prime} \stackrel{g}{\rightarrow} A /\left(A^{\prime}+B^{\prime}\right) \rightarrow 0,
$$

where $f([y])=([y],[y]), g([x],[y])=[x-y]$ and $[\cdot]$ denotes the equivalence class in the appropriate quotient module. We claim that the sequence above is exact.

For its proof we first show that $f$ and $g$ are welldefined homomorphisms. Letting $[y] \in A /\left(A^{\prime} \cap B^{\prime}\right)$ and $x \in A^{\prime} \cap B^{\prime}$, we obtain that

$f([y+x])=([y+x],[y+x])=([y],[y])$. Hence, $f$ is well defined. Moreover, $f$ is a homomorphism, since

$$
\begin{aligned}
f([y]+[z]) & =([y]+[z],[y]+[z]) \\
& =([y],[y])+([z],[z]) \\
f(r[y])= & (r[y], r[y])=r([y],[y]), r \in \mathbf{R} .
\end{aligned}
$$

Similarly, if $([x],[y]) \in A / A^{\prime} \oplus A / B^{\prime}$, and $x_{1} \in A^{\prime}$, $x_{2} \in B^{\prime}$, then

$$
\begin{aligned}
g\left(\left[x+x_{1}\right],\left[y+y_{1}\right]\right) & =\left[\left(x+x_{1}\right)-\left(y+y_{1}\right)\right] \\
& =\left[(x-y)+\left(x_{1}-y_{1}\right)\right]=[x-y],
\end{aligned}
$$

since $x_{1}-y_{1} \in A^{\prime}+B^{\prime}$. Thus, $g$ is well defined.

Moreover, $g$ is a homomorphism, since

$$
\begin{aligned}
& g\left(([x],[y])+\left(\left[x^{\prime}\right],\left[y^{\prime}\right]\right)\right) \\
= & g\left([x]+\left[x^{\prime}\right],[y]+\left[y^{\prime}\right]\right) \\
= & g\left(\left[x+x^{\prime}\right],\left[y+y^{\prime}\right]\right)=\left[\left(x+x^{\prime}\right)-\left(y+y^{\prime}\right)\right] \\
= & {\left[x-y+x^{\prime}-y^{\prime}\right]=[x-y]+\left[x^{\prime}-y^{\prime}\right] }
\end{aligned}
$$

and

$$
\begin{aligned}
g(r([x],[y])) & =g([r x],[r y])=[r x-r y] \\
& =r[x-y], r \in \mathbf{R} .
\end{aligned}
$$

It remains to show that $\operatorname{ker} g=\operatorname{im} f$. For this let $([x],[y]) \in A / A^{\prime} \oplus A / B^{\prime}$ be such that $g([x],[y])=0$. Then $[x-y]=0$, and thus $x-y \in A^{\prime}+B^{\prime}$. This implies that $x+A^{\prime}=y+B^{\prime}$, i.e., $[x]_{A / A^{\prime}}=[y]_{A / B^{\prime}}$ wherefore $\left([x]_{A / A^{\prime}},[y]_{A / B^{\prime}}\right) \in \operatorname{im} f$, and hence $\operatorname{ker} g \subset \operatorname{im} f$.

Conversely, if $([x],[y]) \in \operatorname{im} f$ then $x+A^{\prime}=y+B^{\prime}$ and hence $x+A^{\prime}+B^{\prime}=y+A^{\prime}+B^{\prime}$. It follows that $g([x],[y])=[x-y]=0$ so that $\operatorname{im} f \subset \operatorname{ker} g$. The proof of the claim is complete.

Since $A /\left(A^{\prime}+B^{\prime}\right)$ is a free module, it is in particular projective, and hence the above exact sequence splits (see [4]). Therefore

$$
A / A^{\prime} \oplus A / B^{\prime}=A /\left(A^{\prime} \cap B^{\prime}\right) \oplus A /\left(A^{\prime}+B^{\prime}\right) .
$$

The above equation immediately implies $(\star)$. Now since finite intersection and finite sum of free submodules of $A$, are also free submodules of $A$, a standard use of Mathematical Induction concludes the proof of the theorem.

As every vector space is free over its ground field, the following is an immediate consequence of the above theorem.

Corollary 2.1. If $X$ is a Banach space and $S$ an operator on $X$, for all $M_{i} \in \operatorname{Lat}(S, X), \quad i \in I$

$$
\sum_{i \in I} \operatorname{ind} M_{i}=\operatorname{ind}\left(\bigcap_{i \in I} M_{i}\right)+\operatorname{ind}\left(\sum_{i \in I} M_{i}\right)
$$

In the case where $S$ is a bounded below operator, like the shift operator on Banach spaces of analytic functions, the following, which is the fundamental lemma of this article, holds.

Lemma 2.1. Suppose $M_{i} \in \operatorname{Lat}(S, X), i \in I$, where $S$ is a bounded below operator on a Banach space $X$.

1) We have

$$
\text { ind }\left(\underset{i \in I}{\vee} M_{i}\right) \leq \operatorname{ind}\left(\sum_{i \in I} M_{i}\right) \leq \sum_{i \in I} \operatorname{ind} M_{i} .
$$

2) If $\operatorname{Lat}(S, X)$ contains an invariant subspace of index $m, m \geq 2$ and $n_{i}, \in \mathbb{N} \bigcup\{\infty\}, i \in I$, with $\sum_{i \in I} n_{i}=m$, then there are invariant subspace $N_{i}, i \in I$, such that

$$
\text { ind } N_{i}=n_{i}, i \in I \text {, and ind }\left(\bigvee_{i \in I} N_{i}\right)=\sum_{i \in I} \text { ind } N_{i} \text {. }
$$

Proof. 1) Once more, we shall make use of mathematical induction to prove this corollary. We assume that $M, N \in \operatorname{Lat}(S, X)$ and we show that

$$
\text { ind } M+\text { ind } N \leq \operatorname{ind}(M+N) \leq \text { ind } M+\text { ind } N
$$

(as the initial step of mathematical induction.) If either ind $M$ or ind $N$ is infinite, then there is nothing to prove. So we may assume that ind $M<\infty$ and ind $N<\infty$. Thus there are finite-dimensional subspaces $M_{1}$ and $N_{1}$ of $M$ and $N$, respectively, such that $M=S M+M_{1}, \quad N=S N+N_{1}$, where $\operatorname{dim} M_{1}=\operatorname{ind} M$ and $\operatorname{dim} N_{1}=$ ind $N$. We find that

$$
\begin{aligned}
M+N & =S M+M_{1}+S N+N_{1} \\
& =S(M+N)+M_{1}+N_{1} \\
& \subseteq S(M \vee N)+\left(M_{1}+N_{1}\right) \\
& \subseteq M \vee N .
\end{aligned}
$$

Since $S$ is a bounded below operator, its range is closed (see, e.g., [5], Proposition 6.4, chapter VII), and henceforth the second to last expression as the sum of a closed and a finite-dimensional subspace, it is closed. Since $M+N$ is dense in $M \vee N$ we obtain that the last inclusion in above relations is actually an equality. 
From this it follows that

$$
\begin{aligned}
\operatorname{ind}(M \vee N) & \leq \operatorname{dim}\left(M_{1}+N_{1}\right)=\operatorname{ind}(M+N) \\
& \leq \operatorname{ind} M+\operatorname{ind} N .
\end{aligned}
$$

Now, since the closed span of a finite number of elements in $\operatorname{Lat}(S, X)$ is an element of $\operatorname{Lat}(S, X)$, the proof of (1) follows by mathematical induction.

2) To prove that the equality in 1) can actually occur let us assume that $m \in \mathbb{N} \cup\{\infty\}, m \geq 2$ and that contains an invariant subspace $M$ with index $m$. Without loss of generality let $I=\{1,2,3, \cdots, l\}$,

$n_{1}<n_{2}<n_{3}<\cdots<n_{l}$. At first we assume that

$m=\sum_{i \in I} n_{i}, \quad n_{i} \in \mathbb{N}$. We shall construct

$N_{i} \in \operatorname{Lat}(S, X)$ with ind $N_{i}=n_{i}, i \in I$, and

ind $\left(\vee_{i \in I} N_{i}\right)=m$. As in the proof of part 1) there is an $m$-dimensional subspace $M_{1}$ of $M$ such that

$M=S M+M_{1}$. Let $\left\{g_{1}, g_{2}, \cdots, g_{n_{1}}\right\}$ be a basis for $M_{1}$ and define $N_{1}$ to be the smallest invariant subspace of $S$ which contains $\left\{g_{1}, g_{2}, \cdots, g_{n_{1}}\right\}$. Define $N_{k}$ to be the smallest invariant subspace of $S$ which contains $\left\{g_{n_{k-1}+1}, g_{n_{k-1}+2}, \cdots, g_{n_{k}}\right\}$, where $k=2,3, \cdots, l-1$, and similarly define $N_{l}$ to be the smallest invariant subspace of $S$ which contains $\left\{g_{n_{l-1}+1}, g_{n_{l-1}+2}, \cdots, g_{m}\right\}$. It is easy to observe that $\vee_{i \in I} N_{i}$ is the smallest invariant subspace of $S$ which contains $\left\{g_{1}, g_{2}, \cdots, g_{m}\right\}$.

Claim: ind $N_{1}=n_{1}$

\section{Proof of Claim:}

Let $\mathcal{L}$ be the linear span of $\left\{g_{1}, g_{2}, \cdots, g_{n_{1}}\right\}$. Then $\mathcal{L} \subseteq M_{1}$. We have $M_{1} \cap S M=\{0\}$, thus $S N_{1} \subseteq S M$ implies that $\mathcal{L} \cap S N_{1}=\{0\}$. Furthermore,

$\mathcal{L}+S N_{1} \subseteq N_{1}$ is closed, since $S N_{1}$ is closed and $\mathcal{L}$ is finite dimensional. We note that $\mathcal{L}+S N_{1}$ is invariant for $S$, thus by definition of $N_{1}$ we have

$\mathcal{L}+S N_{1}=N_{1}$. This implies that ind $N_{1}=\operatorname{dim} \mathcal{L}=n_{1} \diamond$

Similarly we see that ind $N_{i}=n_{i}$ for $i=2,3, \cdots, l$ and that ind $\left(\vee_{i \in I} N_{i}\right)=m \diamond$

Finally if $m=\infty$ and $\sum_{i \in I} n_{i}=m$, then there is at least one index $j \in I$ such that $n_{j}=\infty$. If $n_{j}=\infty$ for some $j \in I$, then set $N_{j}=M$. If also

$n_{i}, i \in I \backslash\{j\}=\infty$ then set $N_{i}=M$. In this case we are done because $M=\vee_{i \in I} M$. So suppose that that there is some $j \in I$ such that $n_{j} \in \mathbb{N}$. Since ind $M=\infty$ there is an $n_{j}$-dimensional subspace $M_{j}$ of $M$ such that $S M \cap M_{j}=\{0\}$. Define $N_{j}$ to be the smallest invariant subspace which contains all of $M_{j}$. As in the argument given above it follows that ind $N_{j}=n_{j}$. Clearly, $N_{j} \subseteq M$, thus $M=\vee_{i \in I} N_{i}$ and the proof of part (2) is now complete.

Corollary 2.2. Under the hypothesis of Lemma 2.1 part (2), for the family of $N_{i} \in \operatorname{Lat}(X, S), \quad i \in I$, it holds:

$$
\bigvee_{i \in I} N_{i}=\bigoplus_{i \in I} N_{i}
$$

Proof. To see this, observe that by applying the conclusion of Lemma 2.1 part (1) to the equation of Corollary 2.1 we obtain ind $\left(\bigcap_{i \in I} N_{i}\right)=0$. Hence

$\bigcap_{i \in I} N_{i}=\{0\}$. Therefore by the definition of direct sum (of vector spaces), we get

$$
\bigvee_{i \in I} N_{i}=\bigoplus_{i \in I} N_{i} .
$$

If $S$ is a bounded below operator on $X$ the following is true:

Corollary 2.3. Suppose that $M_{i} \in \operatorname{Lat}(S, X), i \in I$, ind $M_{i}=1$.

If $\bigcap_{i \in I} M_{i} \neq\{0\}$, then strict inequality holds in Lemma 2.1 (a), that is,

$$
\operatorname{ind}\left(\vee_{i \in I} M_{i}\right)<\text { ind }\left(\sum_{i \in I} M_{i}\right) \text {. }
$$

Proof. If $\bigcap_{i \in I} M_{i} \neq\{0\}$, then ind $\left(\bigcap_{i \in I} M_{i}\right) \neq 0$, and since ind $M_{i}=1, i \in I$, then by [2] Theorem 3.16, ind $\left(\bigcap_{i \in I} M_{i}\right)=1$. Thus from Lemma 2.1 (a) and the equation in Corollary 2.1 , we obtain, ind $\left(\vee_{i \in I} M_{i}\right)<\operatorname{ind}\left(\sum_{i \in I} M_{i}\right)$.

The next theorem, which is our main result, follows immediately from Corollary 2.1 and Lemma 2.1, part (1).

Theorem 2.2. if $X$ is a Banach space and $S$ is a bounded below operator, then for $M_{i} \in \operatorname{Lat}(S, X)$, $i \in I \subset \mathbb{N}$,

$$
\operatorname{ind}\left(\sum_{i \in I} M_{i}\right) \geq \operatorname{ind}\left(\bigcap_{i \in I} M_{i}\right)+\operatorname{ind}\left(\bigvee_{i \in I} M_{i}\right) \text {. }
$$

Remark 2.1. We would like to note that [2], Proposition (2.16), Richter proved a special case of our Lemma 2.1 when $S$ is the shift operator on any Banach space $\mathcal{B}$ of analytic functions on an open and connected subset of the complex plane and $m=2$.

Example 2.1. It is well known ([6], Corollary 6.5) that when $S$ is the shift operator on a weighted Bergman space on the unit disk, then for all $m \in \mathbb{N} \cup\{\infty\}$ there are invariant subspaces $N_{m}$, of index $m$. Thus, for this operator Corollary 2.2 applies and we have $\mathrm{V}_{m}=\bigoplus N_{m}$.

\section{REFERENCES}

[1] G. Chailos, "On Reproducing Kernels and Invariant Subspaces of the Bergman Shift," Journal of Operator Theory, Vol. 51, No. 1, 2004, pp. 181-200.

[2] G. Chailos, "Algebraic Properties of the Index of Invariant Subspaces of Operators on Banach Spaces,” Bulletin of the Irish Mathematical Society, Vol. 61, 2008, pp. 913.

[3] J. B. Conway, “A Course in Functionl Analysis,” 2nd Edition, Springer-Verlag, New York, 1990.

[4] H. Hedenmalm, B. Korenblum and K. Zhu, "Theory of 
Bergman Spaces,” Springer-Verlag, New York, 2000. doi:10.1007/978-1-4612-0497-8

[5] T. Hungerford, “Algebra,” Springer-Verlag, New York, 1996.
[6] S. Richter, "Invariant Subspaces in Banach Spaces of Analytic Functions," Transactions of the American Mathematical Society, Vol. 304, No. 2, 1987, pp. 585-616. doi:10.1090/S0002-9947-1987-0911086-8 